\title{
Relations between Aerodynamic Parameters of Heat Transfer and Thermal-Infrared Thermometry in the Bulk Surface Formulation
}

\author{
Dai MATSUSHIMA \\ Department of Geophysics, Tohoku University, Sendai, Japan
}

(Manuscript received 28 June 2004, in final form 1 March 2005)

\begin{abstract}
Disagreement of the thermal-infrared (TIR) surface temperature and the aerodynamic surface temperature, which is an effective surface temperature in estimating surface heat fluxes using the bulk method, is a critical problem in the combination of the TIR thermometry and the bulk method. The present study investigates two methods which adjust the disagreement. One is the method using the radiometric thermal roughness length, and the other is the method using empirical parameterizations (adjustment parameter method). Recent literature has shown a priority of the adjustment parameter method, however, the present study shows the radiometric thermal roughness length is analytically identical to the adjustment parameters, and it is verified by observation data that the two methods can be equally used in estimating sensible heat flux over a rice paddy, over a wide range of vegetation density. Moreover, it is shown that the adjustment parameters quantify the effect of viewing angle of measurement on the TIR surface temperature.
\end{abstract}

\section{Introduction}

Thermal-infrared (TIR) temperature has been used for estimating surface scalar fluxes. There have been many attempts looking for quantitatively accurate methods for the estimations of sensible and latent heat fluxes, which intend to apply them to various types of land cover, especially vegetation and urban surfaces (Kustas and Norman 1996; Voogt and Oke 2003). Of the above attempts, bulk formulations and related Monin-Obukhov similarity theory estimating surface heat fluxes (this is sometimes used with the surface heat budget equations) have been investigated in terms of combined use with TIR surface temperature. In general, an observed TIR surface temperature does not represent the aerodynamic surface tempera-

Corresponding author: Dai Matsushima, Department of Geophysics, Tohoku University, Sendai, 980-8578, Japan.

E-mail: matsu@wind.geophys.tohoku.ac.jp

(C) 2005, Meteorological Society of Japan ture, especially when vegetation canopy is sparse, which reveals a critical problem with regard to estimating sensible heat flux together with the TIR radiometry, namely, the relation between the TIR surface temperature and the aerodynamic surface temperature. The radiometric thermal roughness length $z_{0 h, r}$ is defined according to the TIR surface temperature being substituted for the term of surface temperature in the bulk formulation, as the aerodynamic thermal roughness length $z_{0 h}$ when the aerodynamic surface temperature is substituted. Parameter $z_{0 h}$ has been originally defined in previous literature, which is known as thermal roughness length or aerodynamic roughness length for sensible heat, and shows purely aerodynamic features. In this paper, a similarly defined parameter $z_{0 h, r}$ will play a significant role, and the parameters should be clearly distinguished; Hence $z_{0 h}$ and $z_{0 h, r}$ are named aerodynamic thermal roughness length and radiometric thermal roughness length, respectively. The aerodynamic surface temperature is defined as a hypothetically effective 
temperature for turbulent heat fluxes (sensible heat flux, more specifically) of a certain surface, which is important especially over vegetation and urban canopy, and is often interpreted as the temperature at the height of $z_{0 h}$ on the logarithmic temperature profile in the surface layer (Mahrt et al. 1997; Voogt and Grimmond 2000). Difficulties and their closures in terms of the problem are summarized as follows:

1. The TIR surface temperature (the directional TIR surface temperature measured in a narrow field-of-view) depends on viewing at an oblique angle from the nadir view (Kimes et al. 1980).

2. The sensible heat flux over a vegetation surface is overestimated under unstable conditions in the daytime, when a TIR surface temperature measured in the near nadir angle is substituted for the bulk formulation (Vining and Blad 1992; Matsushima and Kondo 1997).

3. Optimum viewing angles in which a TIR surface temperature agrees with the aerodynamic surface temperature exist according to the leaf area index (LAI) of vegetation (Huband and Monteith 1986; Matsushima and Kondo 1997).

4. Some adjustment schemes should be established in order to estimate the sensible heat flux, when the TIR surface temperature is not measured in the optimum viewing angle. The schemes are divided into two types:

(a) One is the method using the radiometric thermal roughness length, which is adjusted from the aerodynamic thermal roughness length to TIR surface temperature. Using excess resistance revealed in some literature is included in this term (Kustas et al. 1989; Sugita and Brutsaert 1990; Kubota and Sugita 1994),

(b) The other is the method adjusting measured TIR surface temperatures (which is post-corrected in terms of atmospheric absorption, surface emissivity, and device feature) directly to the aerodynamic surface temperature (Chehbouni et al. 1996; Troufleau et al. 1997; Matsushima and Kondo 2000).

5. Radiometric thermal roughness length $z_{0 h, r}$ has been parameterized by solar altitude, LAI, and so on in some cases, but no diurnal and phenological characteritics have been revealed in other cases, and significant parameters contributing to $z_{0 h, r}$ have not been clearly distinguished (Verhoef et al. 1997; Sun et al. 1999).

6. The scheme of adjusting the TIR surface temperature works as a better closure than the radiometric thermal roughness length (Troufleau et al. 1997; Sun et al. 1999), and the key parameters of the scheme depend on LAI (Chehbouni et al. 1996; Matsushima and Kondo 2000).

In this paper, two adjustment paramaters, which adjust a measured TIR surface temperature into the aerodynamic surface temperture, are introduced together with the radiometric thermal roughness length, and they are investigated using a dataset obtained over an almost horizontally homogeneous rice paddy and simulation results of a multi-layer canopy model. The two adjustment parameters have already been proposed in different literature (Chehbouni et al. 1996; Troufleau et al. 1997); however, this study shows that they are essentially the same parameters, by deriving an identical equation connecting the two. Diurnal and phenological variations of parameters with regard to the two schemes are investigated, and distinct variations of these paramaters, even involving the radiometric thermal roughness length are shown.

This paper further aims to reveal formulations relating the two schemes that are derived from the existing bulk formulations and the similarity formulations. The two schemes are shown to be of equivalence in principle, and it was verified that their practical performances are also equivalent within a fairly wide range of LAI, in case of a rice canopy. This discussion is extended to the parameter $k B^{-1}$, which is the logarithm of ratio of the momentum roughness length to the aerodynamic or radiometric thermal roughness length. Some formulations relating the adjustment parameters, the radiometric thermal roughness length, and $k B^{-1}$ are derived to show sensitivites of the contribution of TIR thermometry and aerodynamics to estimates of the sensible heat flux.

At the end of the introduction, a nomenclature of symbols used in this paper is listed as follows: 
$C_{H}$ bulk transfer coefficient for sensible heat $c_{p}$ specific heat of air at constant pressure $d$ zero-plane displacement

$d_{0 h}$ effective height of $T_{0}$

$d_{0 r}$ effective height of $T_{r}$

$h$ canopy height

$k B^{-1}$ a logarithm of ratio of the mometum roughness length to thermal roughness length

$k B_{a}^{-1}$ defined as $k B_{a}^{-1} \equiv \ln \frac{z_{0 m}}{z_{0 h}}$

$k B_{r}^{-1}$ defined as $k B_{r}^{-1} \equiv \ln \frac{z_{0 m}}{z_{0 h, r}}$

$L$ Obukhov length

$T_{0}$ aerodynamic surface temperature

$T_{a}$ air temperature

$T_{c}$ internal canopy surface temperature, $T_{c}(z)$ in case of height from the canopy bottom being specified

$T_{r}$ thermal-infrared surface temperature, $T_{r}(\phi)$ in case of viewing angle $\phi$ from the nadir being specified

$T_{w}$ soil/water surface temperature at the bottom of the canopy

$T_{*}$ friction temperature

$U$ wind speed

$u_{*}$ friction velocity

$z_{0 h}$ aerodynamic thermal roughness length

$z_{0 h, r}$ radiometric thermal roughness length

$z_{0 m}$ aerodynamic momentum roughness length

$\alpha$ adjustment parameter defind as $\alpha \equiv \frac{T_{r}-T_{0}}{T_{r}-T_{a}}$

$\beta$ adjustment parameter defind as $\beta \equiv \frac{T_{0}-T_{a}}{T_{r}-T_{a}}$

$\Delta T_{r}$ surface temperature difference defined as $\Delta T_{r} \equiv T_{r}-T_{0}$

$\zeta$ a normalized height defined as $\zeta \equiv \frac{z}{L}$

$\Psi_{m}(\zeta)$ universal profile function for wind speed as a function of $\zeta$

$\Psi_{h}(\zeta)$ universal profile function for air temperature as a function of $\zeta$

$\Psi_{h, r}(\zeta)$ universal profile function for air temperature as a function of $\zeta$ in case of $z_{0 h}$ being replaced by $z_{0 h, r}$

$\rho$ density of air

\section{Parameterizations}

\subsection{Adjustment parameters}

The sensible heat flux over a vegetation canopy surface is often estimated using the bulk formulation given as

$$
H=c_{p} \rho C_{H} U\left(T_{0}-T_{a}\right),
$$

where $c_{p}$ is the specific heat of air at constant pressure, $\rho$ the density of air, $C_{H}$ the bulk transfer coefficient for sensible heat; $U$ and $T_{a}$ represent the wind speed and air temperature at a reference level, respectively, and $T_{0}$ is the aerodynamic surface temperature. The aerodynamic surface temperature is defined as a representative surface temperature for sensible heat flux under hypothetical isothermal conditions within the canopy regardless of stability, which is regarded as an appropriate temperature to calculate sensible heat flux using the bulk formulation as Eq. (1) (Kondo and Watanabe 1992; Brutsaert and Sugita 1996).

The TIR surface temperature $T_{r}$ measured over a canopy is not, in general, the same as $T_{0}$ (Huband and Monteith 1986; Matsushima and Kondo 1997). The difference between the two temperatures has to be considered when the difference is not negligible, and Eq. (1) is then converted as

$$
H=c_{p} \rho C_{H} U\left(T_{r}-\Delta T_{r}-T_{a}\right),
$$

where $\Delta T_{r}$ is the temperature difference which is defined as $\Delta T_{r} \equiv T_{r}-T_{0}$. If the following equation (with $\alpha$ being constant) is assigned to $\Delta T_{r}$ as

$$
\alpha \equiv \frac{\Delta T_{r}}{T_{r}-T_{a}}=\frac{T_{r}-T_{0}}{T_{r}-T_{a}},
$$

then Eq. (2) will be formulated as

$$
\begin{aligned}
H & =c_{p} \rho C_{H} U\left\{T_{r}-\alpha\left(T_{r}-T_{a}\right)-T_{a}\right\}, \\
& =c_{p} \rho C_{H} U(1-\alpha)\left(T_{r}-T_{a}\right) .
\end{aligned}
$$

The parameter $\alpha$ was originally proposed by Troufleau et al. (1997), in the form $T_{r}-T_{0}=$ $\alpha\left(T_{r}-T_{a}\right)+c$. In this study, however, $c=0$ is assigned, which is justified by the fact that the values of $T_{r}-T_{0}$ will be fairly extrapolated to the original point of the coordinate, as shown in Fig. 1 in the following section.

The other method compensating the lack of the aerodynamic surface temperature in the bulk formulation, when only the TIR surface temperature is available, is formulated as

$$
\begin{aligned}
H & =c_{p} \rho C_{H} U\left(T_{0}-T_{a}\right) \\
& =c_{p} \rho C_{H} U \frac{T_{0}-T_{a}}{T_{r}-T_{a}}\left(T_{r}-T_{a}\right) \\
& =c_{p} \rho C_{H} U \beta\left(T_{r}-T_{a}\right),
\end{aligned}
$$

where $\beta$ is defined as 


$$
\beta \equiv \frac{T_{0}-T_{a}}{T_{r}-T_{a}},
$$

which was originally developed by Chehbouni et al. (1996).

In this study, these two parameters $\alpha$ and $\beta$ are called "adjustment parameters," which means that the temperature differences $T_{r}-T_{0}$ and $T_{r}-T_{a}$ are mainly produced by the viewing angle of a measurement not being set at the optimum viewing angle (Matsushima and Kondo 1997, 2000), and the measured TIR surface temperature should be adjusted to the optimum bulk surface temperature (represented by $T_{0}$ here). This adjustment of the TIR surface temperature can be called "viewing angle correction" (Matsushima and Kondo 2000). This viewing angle effect has been discussed in terms of the radiometric roughness length or the parameter $k B^{-1}$ (Brutsaert and Sugita 1996; Lhomme et al. 2000; Qualls and Yates 2001), and the same explanation will hold for the adjustment parameters, which will be discussed specifically in a later section. The parameters $\alpha$ and $\beta$ denote the rate of the adjustment for the same temperature difference $T_{r}-T_{a}$, which depends on the LAI (Chehbouni et al. 1996; Matsushima and Kondo 2000).

The significant relation between parameters $\alpha$ and $\beta$ is that their sum is equivalent to 1 , that is,

$$
\alpha+\beta=\frac{T_{r}-T_{0}}{T_{r}-T_{a}}+\frac{T_{0}-T_{a}}{T_{r}-T_{a}} \equiv 1 .
$$

\subsection{Radiometric thermal roughness length}

Wind speed $U$ and the temperature difference between the surface and air $T_{0}-T_{a}$ are formulated using the other method when the Monin-Obukhov similarity theory is applied to the bulk formulation in Eq. (1), and these formulations are given as

$$
U=\frac{u_{*}}{k}\left\{\ln \frac{z-d}{z_{0 m}}+\Psi_{m}(\zeta)\right\}
$$

and

$$
T_{0}-T_{a}=\frac{T_{*}}{k}\left\{\ln \frac{z-d}{z_{0 h}}+\Psi_{h}(\zeta)\right\},
$$

where $u_{*}$ is the friction velocity, $T_{*}$ the friction temperature, $k$ the von-Karman constant, $z_{0 m}$ and $z_{0 h}$ are the momentum and aerodynamic thermal roughness length, respectively, $\Psi_{h}(\zeta)$ and $\Psi_{m}(\zeta)$ are the universal profile functions of non-dimensional height $\zeta$, which is composed of height $z-d$ divided by the Obukhov length $L$. Both roughness lengths $z_{0 m}$ and $z_{0 h}$ are aerodynamically defined, because, in the above formulations, the roughness lengths are accompanied only with aerodynamic parameters such as $u_{*}$ and $T_{*}$. The specific forms of the profile function adopted in this study are as follows:

$$
\begin{aligned}
\Psi_{m}(\zeta)= & \ln \frac{\left(x_{m}^{2}+1\right)\left(x_{m}+1\right)^{2}}{\left(x^{2}+1\right)(x+1)^{2}} \\
& +2\left(\tan ^{-1} x-\tan ^{-1} x_{m}\right)
\end{aligned}
$$

and

$$
\Psi_{h}(\zeta)=2 \ln \frac{x_{h}^{2}+1}{x^{2}+1},
$$

where the shear function for the unstable case (Dyer and Hicks 1970) $x=\left(1-16 \frac{z}{L}\right)^{1 / 4}$ is adopted, and $x_{m}=\left(1-16 \frac{z_{0 m}}{L}\right)^{1 / 4}$ and $x_{h}=$ $\left(1-16 \frac{z_{0 h}}{L}\right)^{1 / 4}$. The shear functions for stable conditions are not considered, because this study aims at only studying the unstable conditions in the daytime. The bulk transfer coefficient $C_{H}$ is also formulated by using the similarity theory, and is given as

$$
C_{H}=\frac{k^{2}}{\left(\ln \frac{z-d}{z_{0 m}}+\Psi_{m}(\zeta)\right)\left(\ln \frac{z-d}{z_{0 h}}+\Psi_{h}(\zeta)\right)},
$$

where $d$ is the zero-plane displacement.

If an observed TIR surface temperature $T_{r}$ is applied to the term of the aerodynamic surface temperature $T_{0}$ in Eq. (1), the aerodynamic thermal roughness length should be redefined as the radiometric thermal roughness length, provided the other parameters and variables remain the same. Then, the bulk transfer coefficient with regard to the radiometric thermal roughness length is rewritten as

$$
H=c_{p} \rho C_{H, r} U\left(T_{r}-T_{a}\right),
$$

where

$$
C_{H, r}=\frac{k^{2}}{\left(\ln \frac{z-d}{z_{0 m}}+\Psi_{m}(\zeta)\right)\left(\ln \frac{z-d}{z_{0 h, r}}+\Psi_{h, r}(\zeta)\right)},
$$

where $z_{0 h, r}$ is the radiometric thermal roughness length, $\Psi_{h, r}$ denotes that $z_{0 h}$ is replaced by $z_{0 h, r}$. The radiometric thermal roughness 
length is defined in association with the TIR surface temperature $T_{r}$ in Eq. (13), that is, the combination of $T_{0}$ and $z_{0 h}$ in Eq. (12) is replaced by $T_{r}$ and $z_{0 h, r}$.

\section{Dataset}

The observed dataset used in this study was obtained over an almost horizontally homogeneous rice paddy, located at the Atmospheric Boundary-Layer Observatory of Tohoku University, north of Miyagi Prefecture, Japan $\left(38^{\circ} 33^{\prime} \mathrm{N}, 141^{\circ} 02^{\prime} \mathrm{E}\right)$. The observatory is also surrouneded by a similar rice paddy, having a fetch of more than $2 \mathrm{~km}$ in the directions of the prevailing winds (West-Northwest, Southeast, and Southwest). The surface type of the canopy can be classified into grass or permeable-rough surface based on Brutsaert (1982).

Field experiments investigating the viewing angle distribution of the TIR surface temperture in a narrow field-of-view $\left(2^{\circ}\right)$ over the canopy, sensible heat flux and surface roughness parameters for validation have been carried out during the growing period (from May to September) in 1993 and 1994. For the TIR surface temperature, a spot type infrared thermometer (ER-2007, National) was mounted on a mast at a height of $6.5 \mathrm{~m}$ for viewing at the nadir and several oblique angles $\left(0^{\circ}, 15^{\circ}, 30^{\circ}, 50^{\circ}\right.$, and $70^{\circ}$ from the nadir) on an hourly basis. Three azimuth directions, parallel to the rice row (SouthSouthwest) and at right angles to the row (West-Northwest and East-Southeast), were viewed at each of the nadir oblique angles. In this study, the TIR surface temperatures measured at different azimuth angles are averaged for a representative value of each nadir angle. The TIR surface temperatures measured at $15^{\circ}$ from nadir can be regarded as the temperatures measured at $0^{\circ}$, and are averaged for a representative value of $0^{\circ}$. Each averaged value of the TIR temperature can be regarded as a 30-minute-average, and the justfication is described in Matsushima and Kondo (1997). The data of raw brightness temperature over the canopy were adjusted in terms of the emissivity using the directional sky brightness temperatures observed at the same time. The values of the emissivity are assigned as 0.98 for the cases where the LAI is less than 2, and 0.99 for the cases where the LAI is greater than 2 . These values of emissivity were obtained by additional measurements over a rice canopy using a 1-square-meter acrylic board with a small hole at the center of the board, at which the TIR thermometer views the canopy surface. The emissivity can be roughly estimated by measuring the TIR surface temperatures before and soon after the canopy is covered with the acrylic board, and the TIR sky temperature measured at the same time.

The dataset for the present study is limited to the daytime (0600-1800 h JST) of nine days within the growing period, according to the objective of the present study, which is to investigate the characteristics of phenological and diurnal variations and the mutual relations of the adjustment parameters $(\alpha$ and $\beta$ ) and the radiometric thermal roughness length $z_{0 h, r}$ under unstable conditions. Some data for stable conditions even within the daytime range, are eliminated from the present analysis. There were little cloud and sufficient solar radiation on the nine days, and the wind conditions were relatively mild as much as $6 \mathrm{~ms}^{-1}$ or less. The range of LAI was from 0.04 to 5.4, which covers the overall period of rice canopy growth, and the respective values of LAI are fairly distributed to the growing stages of the phenology. The canopy height and the LAI were measured on the specified nine days, except in the case where the LAI equals 2 , in which the data of two observation days (LAI $=1.6$ and 2.4) were collected because the number of data for the two days was limited. Rice paddies in the growing period are usually fully irrigated, and water is stored over the soil surface at the bottom of the canopy. The depth of water was around $5 \mathrm{~cm}$ on the nine days of present analysis, except on 2 September $1994(\mathrm{LAI}=5.4)$ when no water was stored. Other specifications of the field experiment are referred to Matsushima and Kondo (1997).

For estimating momentum and aerodynamic thermal roughness lengths, zero-plane displacement, and sensible heat flux, a multi-layer numerical model including the vegetation canopy and the atmospheric surface layer, which was based on Kondo and Watanabe (1992), was employed. This model consists of the twostream radiation flux (both shortwave and longwave), a turbulent scheme (equivalent to the level 4 of Mellor-Yamada's scheme without time-variant terms), and momentum drag and 
scalar transfer between the canopy surface and the atmosphere, and the model yields steadystate values of state variables (temperature of atmosphere and canopy surface, atmospheric humidity, and wind speed) and turbulent statistics (fluxes and variances of momentum and scalars) at the end of an iterating calculation. The momentum and scalar fluxes were estimated by optimizing calculated profiles of air temperature, humidity, and wind speed to the observed values, and further, the aerodynamic surface temperature $T_{0}$ is obtained by Eq. (1) using values of $C_{H} U \equiv g_{a}$, which are also calculated by the same numerical model, where $g_{a}$ is the aerodynamic conductance (Matsushima and Kondo 1997). All derived results, such as the two kinds of roughness length, can be regarded as values averaged over 30 minutes, because the meteorological data used in this calculation are 30-minute-averages and the TIR surface temperatures can also be regarded as 30-minute-averages as previously mentioned. The retrieval of $T_{0}$ is based on the concept of Kondo and Watanabe (1992), which agrees to the definition of $T_{0}$ described in Section 2, that is, the sensible heat flux is unchanged, even if the hypothetical condition $T_{c}(z)=T_{g}=T_{0}$ is imposed, where $T_{c}(z)$ is the internal canopy surface temperature at the height of $z$, and $T_{g}$ is the soil/water surface temperature at the bottom of the canopy. This concept is equivalent to an inversion of the bulk formulation for sensible heat. In addition, the momentum and the aerodynamic thermal roughness length and the zero-plane displacement were derived using simulation results of wind speed, air temperature, and the aerodynamic surface temperature. The radiometric thermal roughness length was also calculated in the same way, using the TIR surface temperature instead of the aerodynamic surface temperature. Results of the multi-layer model are regarded as standards, which are compared with the estimated and observed values of fluxes and temperatures appeared in this paper. The root mean squares error of the 30minute-averages of the sensible heat flux is $26 \mathrm{Wm}^{-2}$, compared with the results of both the profile method with the Monin-Obukhov similarity theory and the Bowen ratio method. The error is within the usual observation error, and it looks that adopting one of the three methods is an alternative choice. However, values of sensible heat flux and the residuals of the surface heat budget estimated by the profile and the Bowen ratio methods are not stable in serial on the 30 minutes basis, that is why the multi-layer model is adopted. Values of the aerodynamic roughness length and the zeroplane displacement also agree with the observed estimates derived by Ishida et al. (1997).

\section{Results and discussion}

\subsection{Adjustment parameters $\alpha$ and $\beta$}

The adjustment parameters $\alpha$ and $\beta$ are obtained by the least-squares method using the field data. The values of $\alpha$ and $\beta$ are listed in Table 1 with some parameters characterizing the rice canopy and the correlation coefficient of each regression. Parameters $\alpha$ and $\beta$ are also illustrated in Fig. 1 as the slope of the regression lines. The TIR surface temperatures measured at $0^{\circ}, 30^{\circ}, 50^{\circ}$, and $70^{\circ}$ from the nadir are assigned to the $T_{r}$ term of Eqs. (3) and (6). Systematic differences in TIR surface temperatures according to the viewing angles are found in the case of LAI $=0.42$ (Figs. 1a and 1b). The TIR surface temperatures measured within the range between $0^{\circ}$ and $50^{\circ}$ can be regressed in one line, and the agreement of the regression lines are fairly good. On the other hand, the TIR surface temperatures measured at $70^{\circ}$ should be discriminated from others, and the samples are scattered around the lines $\alpha=0$ and $\beta=1$ (not the regression lines of $70^{\circ}$ ). In the case of LAI $=3.8$ (Figs. $1 \mathrm{c}$ and $1 \mathrm{~d}$ ), the scatterings of the TIR surface temperature differences do not depend on the viewing angles, and they are regressed in lines close to $\alpha=0$ and $\beta=1$. This shows that the TIR surface temperature measured at $70^{\circ}$ from the nadir is not needed to be adjusted to $T_{0}$ in the case of $\mathrm{LAI}=0.42$, which is equivalent to the result of Matsushima and Kondo (1997) that the angle of $70^{\circ}$ from the nadir was near the optimum viewing angle to radiometrically measure the aerodynamic surface temperature over a rice canopy. On the other hand, the TIR surface temperatures can be identified if they are measured within between $0^{\circ}$ and $50^{\circ}$ in this case. The above discrimination is not found and the TIR surface temperature does not depend significantly on the viewing angle in the case of $\mathrm{LAI}=3.8$. Although the scattering of plots 
Table 1. Adjustment parameters $\alpha$ and $\beta$ as a function of leaf area index (LAI) estimated by least-squares method in the format (diurnal average \pm standard error), together with $r$ : correlation coefficient between $T_{r}-T_{a}$ and $T_{r}-T_{0}, z_{0 m}$ : momentum roughness length, $d$ : zero-plane displacement, and $h$ : canopy height.

\begin{tabular}{l|cccc}
\hline LAI & 0.04 & 0.14 & 0.42 & 0.68 \\
\hline$\alpha$ LSM & $0.12 \pm 0.02$ & $0.40 \pm 0.02$ & $0.64 \pm 0.02$ & $0.64 \pm 0.03$ \\
$\beta$ LSM & $0.88 \pm 0.02$ & $0.60 \pm 0.02$ & $0.36 \pm 0.02$ & $0.36 \pm 0.03$ \\
$r$ & 0.50 & 0.87 & 0.97 & 0.88 \\
$z_{0 m}(\mathrm{~m})$ & 0.005 & 0.01 & 0.02 & 0.02 \\
$d(\mathrm{~m})$ & 0.00 & 0.01 & 0.05 & 0.10 \\
$h(\mathrm{~m})$ & 0.10 & 0.15 & 0.16 & 0.18 \\
\hline & 1.0 & 2 & 3.8 & 5.4 \\
& $0.45 \pm 0.03$ & $0.38 \pm 0.03$ & $-0.02 \pm 0.04$ & $-0.20 \pm 0.10$ \\
& $0.55 \pm 0.03$ & $0.62 \pm 0.03$ & $1.02 \pm 0.04$ & $1.20 \pm 0.10$ \\
& 0.68 & 0.86 & -0.00 & -0.11 \\
& 0.03 & 0.05 & 0.07 & 0.13 \\
& 0.13 & 0.2 & 0.3 & 0.4 \\
& 0.21 & 0.3 & 0.55 & 0.85 \\
\hline
\end{tabular}

looks different comparing the figures of $\alpha$ with those of $\beta$, the standard errors of $\alpha$ and $\beta$ in the regression are the same, which is deduced from the identical Eq. (7).

The values of parameter $\alpha$ were calculated in a former study, Matsushima and Kondo (2000), in which the values of the TIR surface temperature measured at $70^{\circ}$ from the nadir, which is close to the optimum viewing angle, were assigned to the aerodynamic surface temperature $T_{0}$. In this study, the values of $\alpha$ are recalculated using the aerodynamic surface temperature $T_{0}$. The present values of $\alpha$ are not significantly different from the corresponding former values over the range of LAI considered here.

Figure 2 shows the parameters $\alpha$ and $\beta$ as a function of LAI. The average values of $\alpha$ are negative in the cases where LAI $=3.8$ and $\mathrm{LAI}=5.4$. This is because the aerodynamic surface temperature was larger than the TIR surface temperature in these cases, which was observed to be generally opposite to the other LAI cases in the daytime. The vertical profile of the internal canopy surface temperature was such that the temperature of the canopy top was higher than the bottom in the above two cases, which was caused by the leaf area density of the canopy along the direct solar path was such a degree that the solar incidence could not warm the bottom of the canopy as the solar zenith angle increases, while a TIR thermometer could detect the temperature of the canopy bottom, as the viewing angle decreases to the nadir because the rice canopy was fairly erectophile. On the other hand, average values of $\beta$ are ranged between 0.3 and 1.2 , which strictly agrees with the identical Eq. (7). Chehbouni et al. (1996) proposed a parameterization of $\beta$ with $L A I$ as

$$
\beta=\frac{1}{\exp \{l /(l-L A I)\}-1},
$$

where the parameter $l$ is set to be a value of 1.5. Equation (15) is valid for LAI ranging between 0.05 and 1 over a sparsely vegetated surface. In the present study, Eq. (15) is not valid for the overall range of LAI considered here, although an exponential function can just fit qualitatively for LAI ranging between 0.1 and 0.5. For the LAI being more than 0.5 , the value of $\beta$ increases as that of $\alpha$ decreases, which the parameterization produced by Chehbouni et al. (1996) can not even qualitatively reproduce. The curve of Eq. (15) is also displayed in Fig. 2.

Next, relations between the parameter $\alpha$, and the TIR and the aerodynamic surface temper- 

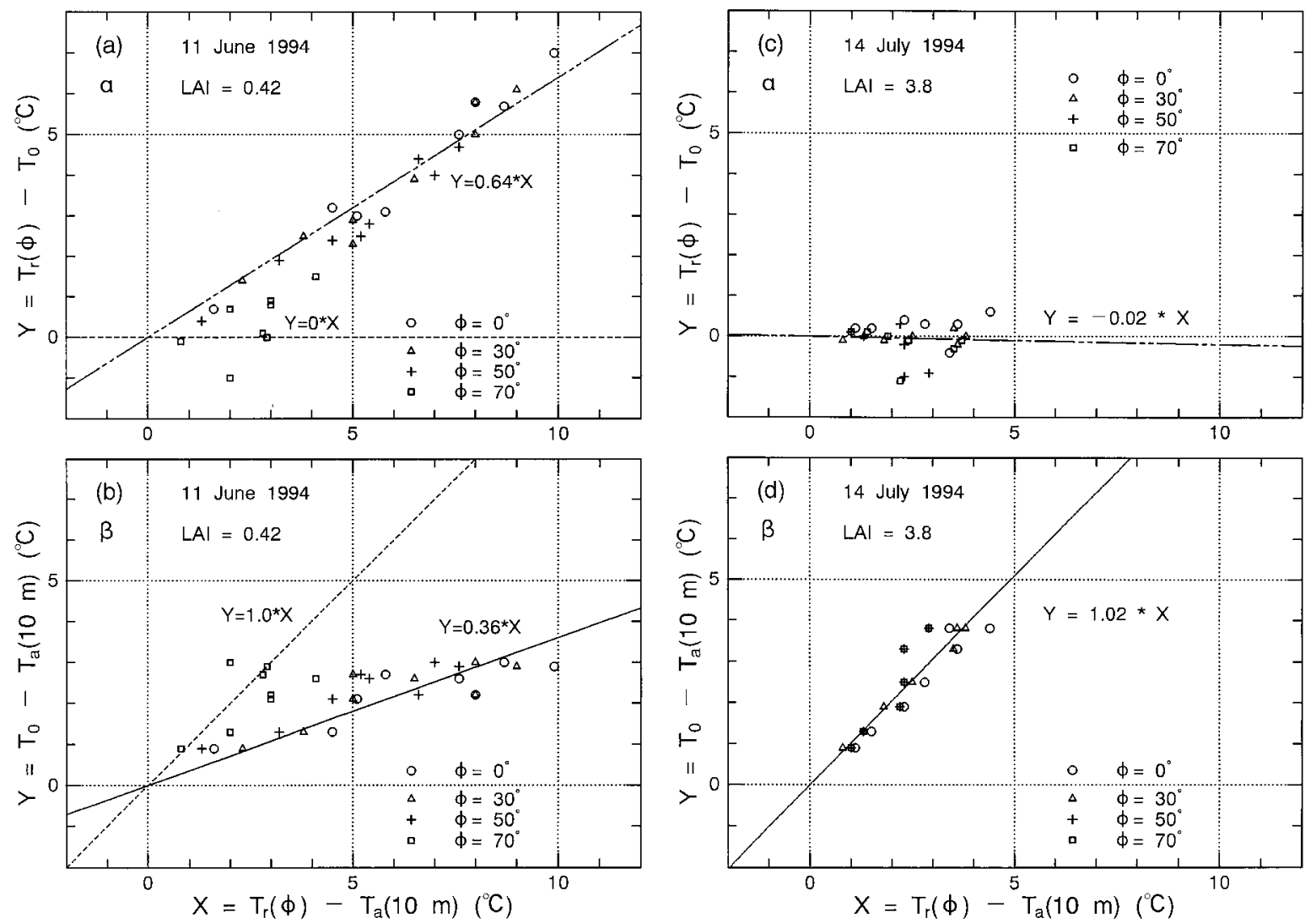

Fig. 1. Difference between the thermal-infrared (TIR) surface temperature and the aerodynamic surface temperature $T_{r}(\phi)-T_{0}(\mathrm{a}, \mathrm{c})$ or that between $T_{0}$ and the air temperature at the height of $10 \mathrm{~m} T_{0}-T_{a}(\mathrm{~b}, \mathrm{~d})$ as a function of $T_{r}-T_{a}$ in cases of LAI $=0.42(\mathrm{a}, \mathrm{b})$ and LAI $=3.8(\mathrm{c}, \mathrm{d})$, where $\phi$ is viewing angle from the nadir. The proportional coefficients of the regression lines are the adjustment parameters $\alpha$ (a, c) and $\beta$ (b, d), except for $\alpha=0$ (a) and $\beta=1$ (b).

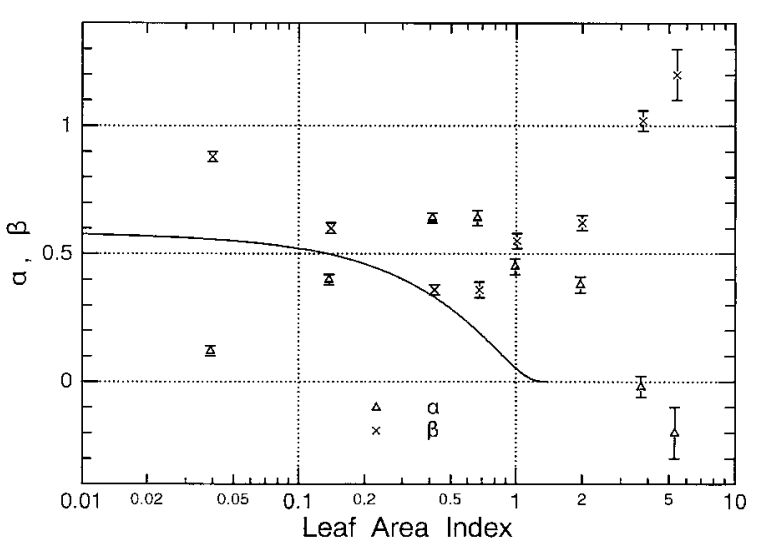

Fig. 2. Parameters $\alpha$ and $\beta$ estimated by LSM as a function of LAI. The curve of the empirical parameterization for $\beta$ by Chehbouni et al. (1996) is also displayed. atures is investigated, and how the parameter $\alpha$ depends on the internal structure of canopy surface temperature is shown. Here, let the effective heights of the aerodynamic and the TIR surface temperatures be defined as the heights of the internal canopy surface temperatures, which correspond to those temperatures, respectively. Figure 3 illustrates the aerodynamic and the TIR surface temperatures (in the nadir view) on the profiles of the internal canopy surface temperature $T_{c}$. Here, $T_{0}, T_{a}$ (air temperature), $T_{c}$, and $U$ (wind speed) are simulated by the multi-layer model using the profile of the leaf area density $a$ (referred to Matsushima and Kondo 1997), and the TIR surface temperature $T_{r}\left(0^{\circ}\right)$ and the temperature at the bottom of the canopy $T_{g}$ are observed. The effective heights and the values of these temperatures are indicated by arrows on the $T_{c}$ profiles. 

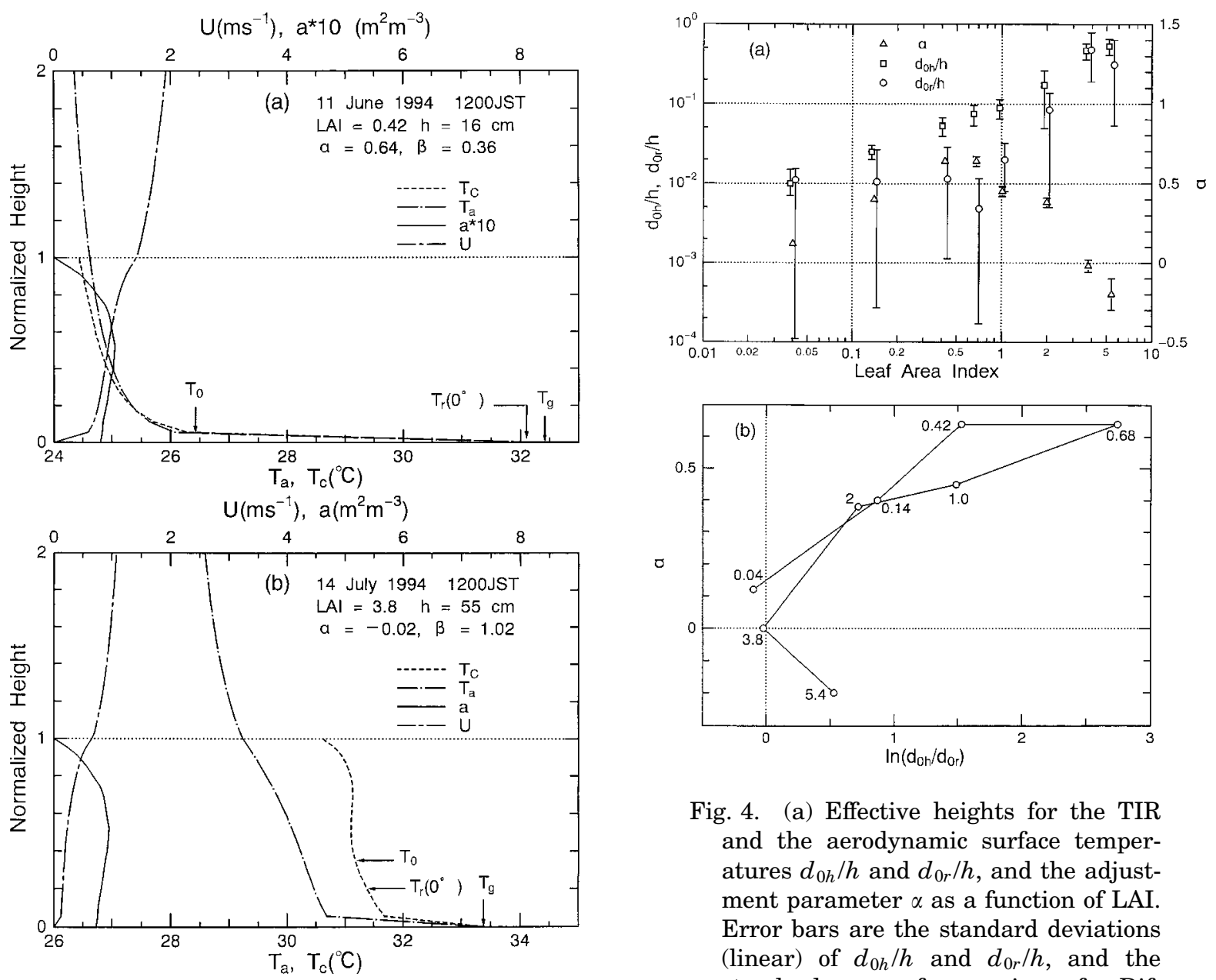

Fig. 4. (a) Effective heights for the TIR and the aerodynamic surface temperatures $d_{0 h} / h$ and $d_{0 r} / h$, and the adjustment parameter $\alpha$ as a function of LAI. Error bars are the standard deviations (linear) of $d_{0 h} / h$ and $d_{0 r} / h$, and the standard errors of regressions of $\alpha$. Different types of symbols for the same LAI are slightly shifted along the horizontal axis from actual locations to be recognized easily. (b) Scattering plots of $\ln \left(d_{0 h} / d_{0 r}\right)$ and $\alpha$. face temperature, $T_{r}\left(0^{\circ}\right)$ the TIR surface temerature measured from the nadir view, $a$ the leaf area density, which is referred to Matsushima and Kondo (1997), $T_{c}$ the internal canopy surface temperature, $T_{a}$ the air temperature, $U$ the wind speed, and $T_{g}$ the soil/water temperature at the canopy bottom.

Comparing Fig. 3a and Fig. 3b, the temperature difference $T_{r}-T_{0}$ increases, and the effective heights of the two temperatures $T_{r}$ and $T_{0}$ move apart from each other, if the value of $\alpha$ increases. Figure $4 a$ shows the normalized effective heights $d_{0 r} / h$ and $d_{0 h} / h$ corresponding to $T_{r}$ and $T_{0}$, respectively, together with the parameter $\alpha$ as a function of LAI, where $h$ is

the canopy height. A high correlation between the two-height difference and $\alpha$ can be observed, and $d_{0 r}$ tends to be separated from $d_{0 h}$ logarithmically, when the LAI is of a moderate amount (approximately $0.2<\mathrm{LAI}<2$ ). Figure $4 \mathrm{~b}$ illustrates this correlation between the logarithmic difference and the paramater $\alpha$, except for $\mathrm{LAI}=5.4$. In the case of $\mathrm{LAI}=5.4, d_{0 r}$ tend to be higher than $d_{0 h}$ in the time other than around noon, which means that $\ln \left(d_{0 h} / d_{0 r}\right)$ is negative, while they are much less than $d_{0 h}$ around noon, the latter leads to $\ln \left(d_{0 h} / d_{0 r}\right)$ being positive on the daytime average. This is because the solar incidence could not penetrate 
the dense rice canopy and warm the bottom of the canopy sufficiently when the solar zenith angle is large, whereas the small solar zenith angles make the condition opposite around noon, which yields that $d_{0 r}$ decreases. The similar samples are found in the cases of $\mathrm{LAI}=0.04$ and $\mathrm{LAI}=3.8$, where the values of $\alpha$ are small. The local minimum of parameter $d_{0 r}$ arises around $0.4-1$, where $\ln \left(d_{0 h} / d_{0 r}\right)$ and the parameter $\alpha$ are both maximum. Otherwise, $d_{0 r}$ and $d_{0 h}$ tend to be in agreement and $\alpha$ is small. Summarizing the above results, the absolute value of $\alpha$ means that the agreement of the effective heights of the TIR surface temperature and the aerodynamic surface temperature over the canopy, and the positive or negative sign of $\alpha$ may denote whether the profile of internal canopy surface temperature increases with height or not, respectively.

\subsection{Radiometric thermal roughness length $z_{0 h, r}$}

The aerodynamic and radiometric roughness lengths $z_{0 h}$ and $z_{0 h, r}$ are calculated on an hourly basis by using Eq. (9), where $z_{0 h}$ and $T_{0}$ are replaced by $z_{0 h, r}$ and $T_{r}$. The values of $T_{r}$ viewed at 0,30 , and $50^{\circ}$ are used in this calculation.

Figure 5 shows the phenological variation of the daytime average of the radiometric thermal roughness length $z_{0 h, r}$, together with the aerodynamic thermal roughness length $z_{0 h}$. The daytime average for roughness lengths used here is a logarithmic average:

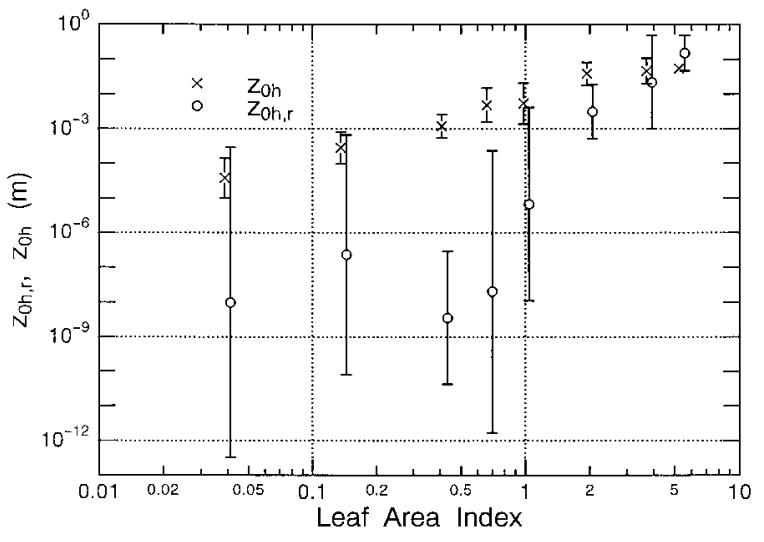

Fig. 5. Phenological variations of the diurnal average of the radiometric thermal roughness length $z_{0 h, r}$, together with the aerodynamic thermal roughness length $z_{0 h}$ as a function of LAI.

$$
\text { (average })=\exp \left\{\overline{\ln \left(z_{0 h} \text { or } z_{0 h, r}\right)}\right\},
$$

which averages (denoted by an overbar) over the diurnal range (0600-1800 h JST). The phenological variations of both $z_{0 h, r}$ and $z_{0 h}$ show almost monotonous increase as LAI increases, except that $z_{0 h, r}$ has a local minimum when the LAI is a moderate amount, and the range of variation of $z_{0 h, r}$ is much larger than that of $z_{0 h}$. Compared to Fig. 4, phenological variations of $z_{0 h}$ and $z_{0 h, r}$ resemble $d_{0 r} / h$ and $d_{0 h} / h$, respectively.

Figure 6 shows the diurnal variations of $z_{0 h, r}$ and $z_{0 h}$ in two different LAI cases. Different features according to LAI can be seen in the diurnal variations of the roughness lengths. For a moderate amount of LAI (shown in Fig. 6a), $z_{0 h, r}$ decreases as a function of time regardless of the viewing angles, while $z_{0 h}$ remains within
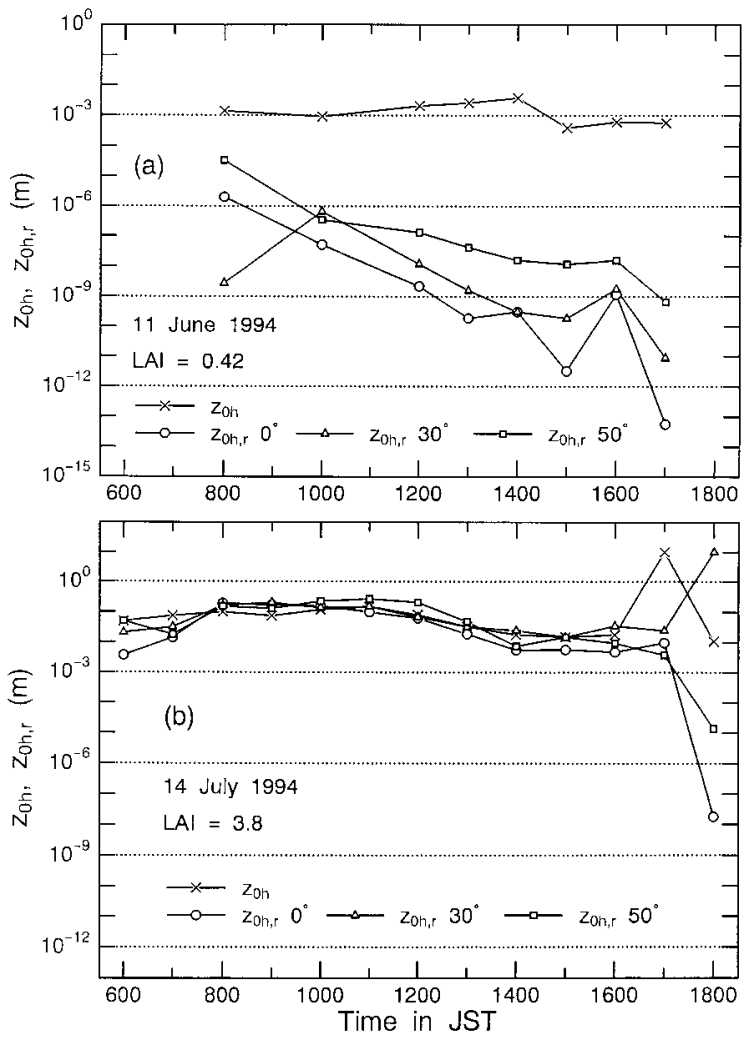

Fig. 6. Diurnal variation of the radiometric thermal roughness length $z_{0 h, r}$ together with the aerodynamic thermal roughness length $z_{0 h}$ in cases of $\mathrm{LAI}=0.42$ (a) and $\mathrm{LAI}=3.8(\mathrm{~b})$. The values of $0^{\circ}, 30^{\circ}$, and $50^{\circ}$ denote the viewing angles of the TIR thermometry. 
a narrow range regardless of the increase in time, and the values are much larger than $z_{0 h, r}$. The daytime averages of $z_{0 h, r}$ and $z_{0 h}$ are close to their original values around noon. On the other hand, for a large value of LAI (shown in Fig. 6b), both $z_{0 h, r}$ and $z_{0 h}$ do not show significant changes, correlate well, and are fairly close to the values of the momentum roughness length $z_{0 m}$, except in the late afternoon (See Table 1 for the values of $z_{0 m}$ ). The dispersion of the values appearing at $1700 \mathrm{~h}$ and $1800 \mathrm{~h}$ is because the mutual differences between $T_{r}, T_{0}$, and $T_{a}$ were very small.

The almost monotonous decrease shown in the case of $\mathrm{LAI}=0.42$ may be attributed to the diuranal variation of the TIR surface temperature. Figure 7 shows the diurnal variation of
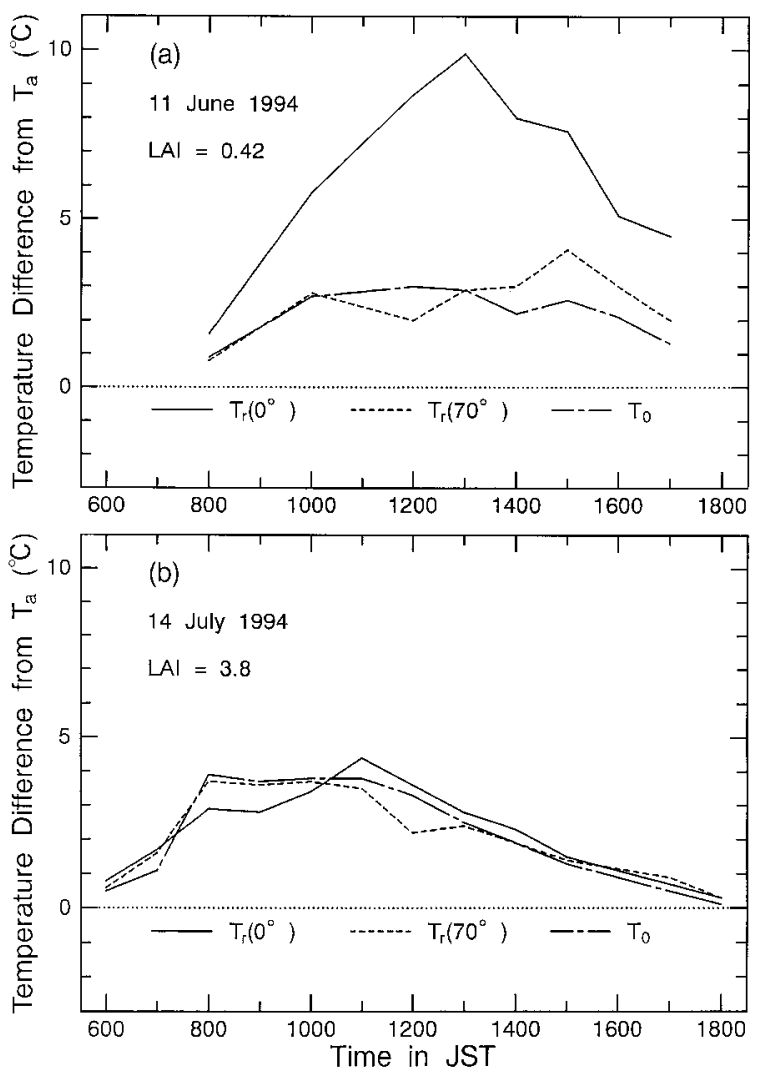

Fig. 7. Diurnal variations of the TIR surface temperature $T_{r}$ viewed at $0^{\circ}$ and $70^{\circ}$ from the nadir together with the aerodynamic surface temperature $T_{0}$ in cases of $\mathrm{LAI}=0.42$ (a) and $\mathrm{LAI}=3.8$ (b). The values are plotted as differences from the air temperature $T_{a}$ at the height of $10 \mathrm{~m}$ for every plot. the TIR surface temperature measured in the nadir direction $T_{r}\left(0^{\circ}\right)$ and the aerodynamic surface temperature $T_{c}$, which are illustrated as differences from the air temperature $T_{a}$. In Fig. 7a, difference between $T_{r}\left(0^{\circ}\right)$ and $T_{c}$ is small in the early morning, whereas the difference enlarges in the late afternoon. The magnitudes of sensible heat flux are almost the same in both times (not shown), and so is the difference $T_{0}-T_{a}$, whereas the difference $T_{r}\left(0^{\circ}\right)-T_{a}$ in the late afternoon is larger than that in the early morning, which gets the radiometric thermal roughness length smaller in the late afternoon. The high TIR surface temperature may be caused by the surface temperature of water storage at the bottom of the rice canopy, which remains warm even in the late afternoon because of the large heat capacity of water. Moreover, the TIR thermometer at the nadir view can easily detect the warm surface because the leaf area index is small, compared to that viewing at $70^{\circ}$ from the nadir, which detects more leaf surface. On the other hand, in Fig. 7b, both the differences correlate well with each other, which does not cause a significant difference between the two thermal roughness lengths. The conditions in this case are different from the other: the water surface is not warmed sufficiently, and the leaf area index is large.

\subsection{Mutual relations of parameters $z_{0 h, r}, \alpha, \beta$, and $k B^{-1}$}

Rearranging the terms of Eq. (4) with Eq. (13) yields

$$
C_{H, r}=C_{H}(1-\alpha) .
$$

Starting from Eq. (17), a formulation between $\alpha$ and $z_{0 h, r}$ is obtained using Eqs. (12) and (14) as follows:

$$
\begin{aligned}
\alpha & =1-\frac{\ln \frac{z-d}{z_{0 h}}+\Psi_{h}(\zeta)}{\ln \frac{z-d}{z_{0 h, r}}+\Psi_{h, r}(\zeta)}, \\
& =\frac{\ln \frac{z_{0 h}}{z_{0 h, r}}+2 \ln \frac{x_{h, r}^{2}+1}{x_{h}^{2}+1}}{\ln \frac{z-d}{z_{0 h, r}}+2 \ln \frac{x_{h, r}^{2}+1}{x^{2}+1}}, \\
& \sim \frac{\ln \frac{z_{0 h}}{z_{0 h, r}}}{\ln \frac{z-d}{z_{0 h, r}}+2 \ln \frac{x_{h, r}^{2}+1}{x^{2}+1}} .
\end{aligned}
$$


The approximation of the third line of Eq. (18) is derived from the result that the value of the term $2 \ln \frac{x_{h, r}^{2}+1}{x_{h}^{2}+1}$ is practically almost negligible. Considering the values of each term in Eq. (18) under practical conditions, the first term of the denominator $\left(\ln \frac{z-d}{z_{0 h, r}}\right)$ is much larger than the second term of the denominator by one order. Then, the second term can be eliminated from Eq. (18), which is given as

$$
\alpha \sim\left(\ln \frac{z_{0 h}}{z_{0 h, r}}\right) /\left(\ln \frac{z-d}{z_{0 h, r}}\right),
$$

which denotes that the atmospheric stability of the surface layer represented by $(z-d) / L$ is not needed in considering thermal roughness parameters and the adjustment parameters.

Equation (19) can be expressed using the momentum roughness $z_{0 m}$ as

$$
\begin{aligned}
\alpha & \sim \frac{\ln \frac{z_{0 m}}{z_{0 h, r}}-\ln \frac{z_{0 m}}{z_{0 h}}}{\ln \frac{z_{0 m}}{z_{0 h, r}}+\ln \frac{z-d}{z_{0 m}}}, \\
& =\frac{k B_{r}^{-1}-k B_{a}^{-1}}{k B_{r}^{-1}+\ln \frac{z-d}{z_{0 m}}} .
\end{aligned}
$$

Here, $k B_{a}^{-1} \equiv \ln \frac{z_{0 m}}{z_{0 h}}$ and $k B_{r}^{-1} \equiv \ln \frac{z_{0 m}}{z_{0 h, r}}$, which can be called as "aerodynamic" and "radiometric" $k B^{-1}$ parameters, respectively, are assigned.

The identical Eq. (7) shows similar results regarding $\beta$ as

$$
\beta \sim 1-\left(\ln \frac{z_{0 h}}{z_{0 h, r}}\right) /\left(\ln \frac{z-d}{z_{0 h, r}}\right),
$$

and

$$
\beta=\frac{k B_{a}^{-1}+\ln \frac{z-d}{z_{0 m}}}{k B_{r}^{-1}+\ln \frac{z-d}{z_{0 m}}} .
$$

Thus, the parameters $\alpha$ and $\beta$ finally link the "aerodynamic" and "radiometric" $k B^{-1}$ parameters.

In order to verify the approximation of Eq. (19), values of $\alpha$ obtained by Eqs. (18) and (19) are compared to values of $\alpha$ derived from Eq. (3) (by definition) in Fig. 8a on an hourly basis. This figure illustrates that both Eqs. (18) and (19) are valid in estimating $\alpha$, if the value of $\alpha$ is positive. Although results of Eq. (19) tend to differ from those of Eqs. (3) and (18) when values of $\alpha$ are negative, values of $T_{r}-T_{a}$ tend
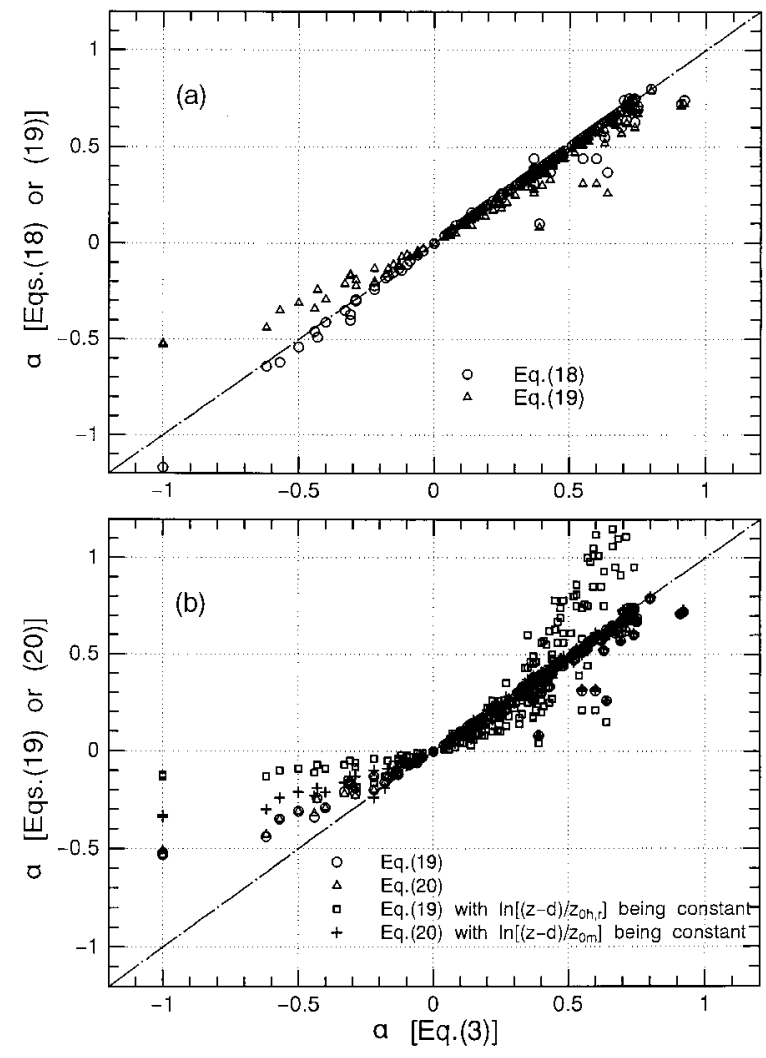

Fig. 8. (a) Estimates of the adjustment parameter $\alpha$ calculated by Eqs. (18) and (19) compared with values of $\alpha$ calculated by the definition (Eq. [3]). (b) Sensitivity of Eqs. (19) and (20) illustrated by a change of $\alpha$.

to be so small in this case, in which LAI is larger than approximately 4 , that sensitivity of $\alpha$ to the rate of adjustment becomes weak. As a consequence, Eq. (19) is valid as a reasonable approximation for estimating $\alpha$.

Further, investigating Eqs. (19) and (20), the sensitivity of aerodynamics and TIR thermometry to $\alpha$ can also be found. Figure 8b shows the sensitivity of $\alpha$ estimated by Eq. (19) when the term $\ln \frac{z-d}{z_{0 h, r}}$ is assigned by a constant, which is an approximate average value over the growing period of rice. The denominator of Eq. (19) $\ln \frac{z-d}{z_{0 h, r}}\left(\sim k \frac{T_{r}-T_{a}}{T_{*}}\right)$ expresses how much the temperature difference $T_{r}-T_{a}$ is effective to the sensible heat represented by an aerodynamic parameter $T_{*}$, where $T_{r}$ is measured at $0-50^{\circ}$ from the nadir. From the discussion with regard to Figs. 6 and 7, when the LAI is small, 
$T_{r}-T_{a}$ is only slightly effective in aerodynamics, and hence to $T_{*}$, whereas solar radiation and TIR thermometry play an important role in realizing $T_{r}$. However, these features become inconspicuous as the LAI increases. Therefore, a significant change in values of Eq. (19) appears after the assignment. On the other hand, the sensitivity of $\alpha$ estimated by Eq. (20) is also illustrated in the Fig. 8b; the term $\ln \frac{z-d}{z_{0 m}}$ is assigned by an approximate average value over the growing period of rice, and no significant change appears, especially when values of $\alpha$ are positive. Similarly, the term $\ln \frac{z-d}{z_{0 m}}\left(\sim k \frac{U}{u_{*}}\right)$ in Eq. (20) expresses how much wind speed $U$ is effective to the momentum flux represented by $u_{*}$. Parameter $u_{*}$ is proportional to $U$, provided the momentum roughness length is unchanged and the stability change is not very significant in theory. These conditions are realized in the respective days of the present analysis. Similar sensitivity tests show that the most sensitive parameter in Eq. (20) is $k B_{r}^{-1}$, which is much more sensitive than $k B_{a}^{-1}$ and $\ln \frac{z-d}{z_{0 m}}$ (not shown in the figures), which coincides with the calculated result that $k B_{r}^{-1}$ depends significantly on the LAI and the viewing angle (Lhomme et al. 2000). Therefore, parameter $\alpha$ is reasonably interpreted as an explicit indicator of the viewing angle effect concerned with the TIR thermometry, and $\alpha$ multiplied by $T_{r}-T_{a}$ is the amount that is to be reduced in order to obtain the aerodynamic surface temperature from a measured TIR surface temperature. This operation of temperature reduction can be termed as "viewing angle correction."

Figure 9 shows diurnal averages and the standard deviations (on an hourly basis) of $\alpha$ as a function of LAI estimated by six methods of calculation, which are [1] least-squares method (LSM), [2] diurnal averages of $z_{0 h}$ and $z_{0 h, r}$ calculated by Eq. (16), which is assigned to Eq. (19), [3] Eq. (3), [4] Eq. (18), [5] Eq. (19), and [6] Eq. (20) (See Table 2 for specific values). The diurnal averages of $\alpha$ are such that the maximum likelihood estimates of the LSM for the method [1], and the averages over respective daytimes for the methods [2]-[6]. Results converge fairly well for each value of LAI regardless of the choice of the methods, except where LAI is 0.04 and 5.4. The dispersion of values for $\mathrm{LAI}=5.4$ case is due to the results that tem-

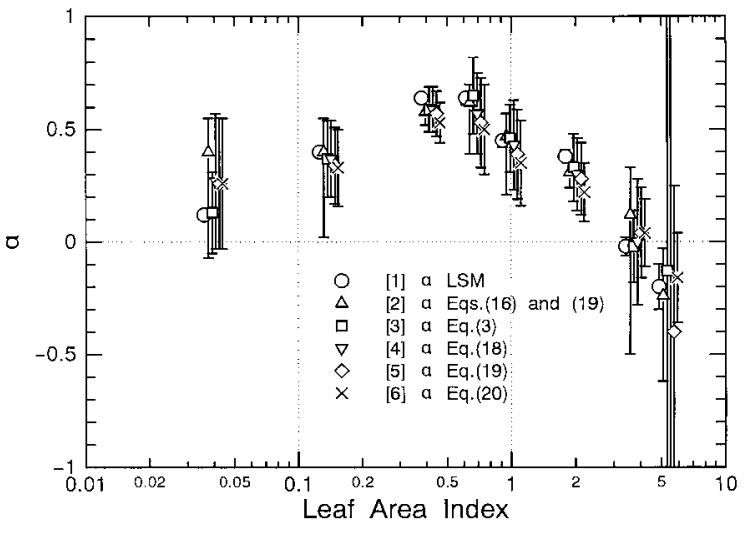

Fig. 9. Estimates of $\alpha$ by six methods of calculation as a function of LAI. Explanation of captions described in text. The symbols denote the diurnal averages, and the error bars denote standard deviations or a standard error in daytime. Locations of symbols are shifted horizontally, as in Fig. 4.

perature difference $T_{r}-T_{a}$ is so small that resulted $\alpha$ tend to fluctuate. The result shows that the estimates of the rates of the viewing angle correction are almost mutually equivalent regardless of the choice of the methods in the case of rice paddy.

There have been many attempts to formulate the radiometric thermal roughness length $z_{0 h, r}$ as functions of LAI, solar radiation, wind speed, and some other parameters. However, those attempts have been concluded to be difficult for an appropriate formulation of $z_{0 h, r}$, and using $z_{0 h, r}$ is not an operational way to assign the TIR surface temperature to the bulk formulation for estimating the sensible heat flux (Troufleau et al. 1997; Verhoef et al. 1997; Sun et al. 1999; Qualls and Yates 2001; Mahrt and Vickers 2004) except some earlier studies (Kustas et al. 1989; Sugita and Brutsaert 1990). In this study, on the case of the rice paddy, the diurnal variation of $z_{0 h, r}$ is almost monotonous, logarithmically decreasing as illustrated in Fig. 6a, besides the diurnal average is close to the value around noon, when the LAI is less than about 2 . When the LAI is larger, diurnal variation of $z_{0 h, r}$ is not significant and the values are close to $z_{0 h}$. This is caused by variations of the leaf and water surface temperature within the canopy, and specifically, the monotonous decrease 
Table 2. Adjustment parameters $\alpha$ as a function of LAI estimated by six methods of calculation listed in the same format as Table 1 except for $\alpha$ Eqs. (16) and (19) represented as (average subtracted by logarithmic standard error-average-average added by logarithmic standard error). See text for calculation methods.

\begin{tabular}{l|cccc}
\hline LAI & 0.04 & 0.14 & 0.42 & 0.68 \\
\hline$\alpha$ LSM & $0.12 \pm 0.02$ & $0.40 \pm 0.02$ & $0.64 \pm 0.02$ & $0.64 \pm 0.03$ \\
$\alpha$ Eq. (3) & $0.13 \pm 0.18$ & $0.37 \pm 0.17$ & $0.59 \pm 0.10$ & $0.65 \pm 0.17$ \\
$\alpha$ Eqs. (16) and (19) & $-0.07-0.40-0.55$ & $0.02-0.40-0.55$ & $0.52-0.58-0.63$ & $0.39-0.62-0.70$ \\
$\alpha$ Eqs. (18) & $0.27 \pm 0.30$ & $0.37 \pm 0.17$ & $0.59 \pm 0.10$ & $0.57 \pm 0.18$ \\
$\alpha$ Eq. (19) & $0.26 \pm 0.29$ & $0.34 \pm 0.17$ & $0.59 \pm 0.10$ & $0.53 \pm 0.20$ \\
$\alpha$ Eq. (20) & $0.26 \pm 0.29$ & $0.33 \pm 0.17$ & $0.53 \pm 0.09$ & $0.50 \pm 0.20$ \\
\hline & 1.0 & 2 & 3.8 & 5.4 \\
\hline & $0.45 \pm 0.03$ & $0.38 \pm 0.03$ & $-0.02 \pm 0.04$ & $-0.20 \pm 0.10$ \\
& $0.46 \pm 0.15$ & $0.33 \pm 0.15$ & $-0.02 \pm 0.16$ & $-0.13 \pm 1.75$ \\
& $0.21-0.47-0.57$ & $0.24-0.31-0.36$ & $-0.50-0.12-0.33$ & $-0.62--0.24--0.03$ \\
& $0.43 \pm 0.20$ & $0.30 \pm 0.16$ & $0.00 \pm 0.28$ & $-1.05 \pm 2.42$ \\
& $0.39 \pm 0.20$ & $0.28 \pm 0.16$ & $0.04 \pm 0.20$ & $-0.40 \pm 0.65$ \\
& $0.35 \pm 0.19$ & $0.22 \pm 0.13$ & $0.04 \pm 0.20$ & $-0.16 \pm 0.20$ \\
\hline
\end{tabular}

of $z_{0 h, r}$ may be due to the result that the water surface temperature in the late afternoon is higher than in the early morning, even when the aerodynamic surface temperatures are the same levels at both times.

Figure 10 shows the diurnal average and the standard deviation (on an hourly basis) of errors in estimating the sensible heat flux using

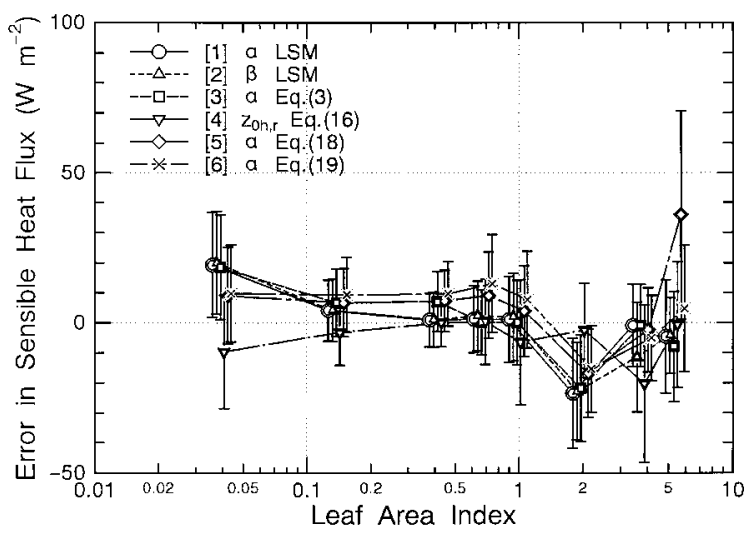

Fig. 10. Errors in estimates of the sensible heat flux using the diurnal averages of $\alpha$ estimated by the six methods of calculations. Explanation of captions described in text. The symbols denote the diurnal averages of error and error bars denote standard deviations. The locations of symbols are shifted horizontally, as in Fig. 4. six different methods of calculation, using each of the three parameters $\left(\alpha, \beta\right.$, and $\left.z_{0 h, r}\right)$ assigned in the bulk formulation. The parameters used in the six methods of calculation are as follows: [1] $\alpha$ derived by the LSM, [2] $\beta$ derived by the LSM, [3] $\alpha$ calculated by Eq. (3), [4] $z_{0 h, r}$ estimated by Eq. (16), [5] $\alpha$ estimated by Eq. (18), and [6] $\alpha$ estimated by Eq. (19), respectively (See Table 3 for specific values). The error of the sensible heat flux is defined as the difference from the corresponding estimate by the multi-layer model, and the magnitudes of the sensible heat flux are approximately between $0-120 \mathrm{Wm}^{-2}$. The calculation of diurnal averages and standard deviations are the same as in Fig. 9. The magnitutes of the error of the diurnal average are almost within $20 \mathrm{Wm}^{-2}$ regardless of the LAI, and the standard deviations converge within $20 \mathrm{Wm}^{-2}$ in most cases, which is compared with the typical error of observation. The dispersion of $z_{0 h, r}$ for small values of LAI does not so influence both the averages and the standard deviations, because absolute values of temperature difference $T_{r}-T_{a}$ in the early morning and the late afternoon are usually small; hence, the same applies for sensible heat flux. No significant difference is found between cases of the adjustment parameters ([1], [2], and [3]) and the radiometric thermal roughness length ([4]). Results of the methods [5] and [6], which use 
Table 3. Error in estimating the sensible heat flux as a function of LAI estimated by six methods of calculation in the same form as Table 1. See text for calculation methods.

\begin{tabular}{l|rccccccc}
\hline LAI & \multicolumn{1}{|c}{0.04} & 0.14 & 0.42 & 0.68 & 1.0 & 2 & \multicolumn{1}{c}{3.8} & 5.4 \\
\hline$\alpha$ LSM & $19 \pm 17$ & $4 \pm 10$ & $1 \pm 9$ & $1 \pm 11$ & $1 \pm 14$ & $-23 \pm 18$ & $-1 \pm 14$ & $-5 \pm 19$ \\
$\beta$ LSM & $20 \pm 17$ & $4 \pm 10$ & $1 \pm 9$ & $2 \pm 12$ & $2 \pm 15$ & $-23 \pm 16$ & $-11 \pm 18$ & $-4 \pm 13$ \\
$\alpha$ Eq. (3) & $19 \pm 17$ & $7 \pm 11$ & $7 \pm 10$ & $0 \pm 11$ & $0 \pm 14$ & $-22 \pm 18$ & $-1 \pm 14$ & $-8 \pm 18$ \\
$z_{0 h, r}$ Eq. (16) & $-10 \pm 19$ & $-3 \pm 11$ & $0 \pm 8$ & $0 \pm 14$ & $-6 \pm 21$ & $-2 \pm 16$ & $-20 \pm 26$ & $-1 \pm 21$ \\
$\alpha$ Eq. (18) & $9 \pm 16$ & $7 \pm 11$ & $7 \pm 10$ & $9 \pm 14$ & $4 \pm 15$ & $-17 \pm 15$ & $-2 \pm 14$ & $36 \pm 35$ \\
$\alpha$ Eq. (19) & $10 \pm 16$ & $9 \pm 12$ & $10 \pm 10$ & $13 \pm 16$ & $8 \pm 16$ & $-15 \pm 15$ & $-5 \pm 14$ & $5 \pm 21$ \\
\hline
\end{tabular}

values of $\alpha$ converted from $z_{0 h, r}$, also denote no significant differences from other cases. The above results verify the equivalence of the adjustment parameters and the radiometric thermal roughness length, which link the aerodynamic and the TIR surface temperatures in rice canopy cases.

\section{Summary and conclusion}

Two different parameterizations which should be implemented alternatively into the bulk formulation for estimating sensible heat flux are investigated using observed data over a rice canopy and corresponding simulation results calculated with a multi-layer canopy model. The LAI of the rice paddy investigated in this study ranges from 0.04 to 5.4, which covers the overall range of growing stages of rice canopy and corresponds to the grass or the permeable rough surface in terms of aerodynamics designated in Brutsaert (1982).

One of the parameterizations is the radiometric thermal roughness length $z_{0 h, r}$, which is defined similarly as the aerodynamic thermal roughness length, when a TIR surface temperature measured at a near nadir angle $T_{r}$ is assigned to the term of surface temperature in the bulk formulation. The other one is the adjustment parameters $\alpha$ and $\beta$, which should be alternatively used. Parameter $\alpha$ is defined as a ratio of the difference between the TIR surface temperature and the aerodynamic surface temperature $T_{r}-T_{0}$ to the difference between the TIR surface temperature and the air temperature $T_{r}-T_{a}$. Parameter $\beta$ is similarly defined as $\alpha$, and both the parameters are connected by the identical equation, $\alpha+\beta \equiv 1$.

Parameter $\alpha$ has the maximum value when LAI is approximately 0.5 , which corresponds to $\beta$ having the minimum value. The variation of $\alpha$ as a function of LAI is correlated well with the variation of the difference between the normalized effective height of the aerodynamic surface temperature $d_{0 h} / h$ and the TIR surface temperature $d_{0 r} / h$, except for the case of LAI $=5.4$. These two heights $d_{0 h} / h$ and $d_{0 r} / h$ are highly correlated with $z_{0 h}$ and $z_{0 h, r}$, respectively. The variation of $\beta$ as a function of LAI does not agree with an empirical parameterization proposed by Chehbouni et al. (1996). The logarithmic diurnal average of the thermal roughness lengths $z_{0 h}$ and $z_{0 h, r}$ is found to be a function of LAI: the former shows monotonous increase and the latter shows abrupt decrease when the LAI is of a moderate amount (approximately $0.2-2$ ). The diurnal variation of $z_{0 h, r}$ on an hourly basis shows an almost monotonous decrease in time series, especially for the values when LAI is of a moderate amount. This ruled variation in time series has not been confirmed in previous literature, and may be due to the diurnal variation of water surface temperature at the canopy bottom, which is influenced by the findings that the canopy structure controls aerodynamics and the solar incidence within the canopy, and the TIR thermometry viewing over the canopy.

Mutual relations between parameters $\alpha, \beta$, $z_{0 h, r}$, and $k B^{-1}$ are formulated, and the formulations are well verified with observed data. Estimated values of $\alpha$ using the six different methods of calculation converge, except when LAI is rather small or large. Errors in estimating the sensible heat flux also converge well within about $20 \mathrm{Wm}^{-2}$ as a standard error, over the daytime regardless of LAI and the parameters being used. Sensitivity tests confirm the principal meaning of parameter $\alpha$ (and $\beta$ ). These parameters function as eliminating the component of the temperature difference be- 
tween the surface and the air, which is not effective to the sensible heat flux and is arised by a measurement of TIR surface temperature at an inappropriate viewing angle. Therefore, the function of parameters $\alpha$ and $\beta$ can be termed as "viewing angle correction." The present study describes that some parameters concerned with connection of TIR thermometry and the aerodynamic parameters in estimating the sensible heat flux using the bulk formulation are mutually connected in some relatively simple formulations without significant errors in a rice canopy case. Diurnal and phenological variations of the radiometric thermal roughness length $z_{0 h, r}$ have not always been confirmed in previous literature (Verhoef et al. 1997; Mahrt and Vickers 2004). In this study, formulations and distinct empirical relations with regard to $z_{0 h, r}$ are derived and verified by an observation dataset, which was obtained over a rice canopy where water was stored at the bottom. Formulations derived in this study need to be verified for other land covers to generalize the results obtained here.

\section{Acknowledgments}

The author thanks Dr. T. Hiyama of Nagoya University for reviewing an earlier version of this manuscript. Thanks are also extended to two anonymous reviewers who provided many valuable comments. This study was partly supported by a Grant-in-Aid for Scientific Research by the Ministry of Education, Science, Culture and Sports of Japan (No. 13740274).

\section{References}

Brutsaert, W., 1982: Evaporation into the atmosphere. D. Reidel Publishing Company, 299pp. and M. Sugita, 1996: Sensible heat transfer parameterization for surfaces with anisothermal dense vegetation. J. Atmos. Sci., 53, 209-216.

Chehbouni, A., D.L. Seen, E.G. Njoku, and B.M. Monteny, 1996: Examination of the difference between radiative and aerodynamic surface temperatures over sparsely vegetated surfaces. Remote Sens. Environ., 58, 177-186.

Dyer, A.J. and B.B. Hicks, 1970: Flux-gradient relationships in the constant flux layer. Quart. J. Roy. Meteor. Soc., 96, 715-721.

Huband, N.D.S. and J.L. Monteith, 1986: Radiative surface temperature and energy balance of a wheat canopy, I: Comparison of radiative and aerodynamic canopy temperature. Bound.Layer Meteor., 36, 1-17.

Ishida, S., T. Ishida, and J. Kondo, 1997: The seasonal variation of heat balance on a paddy field. J. Japan Soc. Hydrology \& Water Resources, 10, 123-132 (in Japanese with English abstract).

Kimes, D.S., S.B. Idso, J.P.J. Pinter, R.J. Reginato, and R.D. Jackson, 1980: View angle effects in the radiometric measurement of plant canopy temperatures. Remote Sens. Environ., 10, 273284.

Kondo, J. and T. Watanabe, 1992: Studies on the bulk transfer coefficients over a vegetated surface with a multilayer energy budget model. $J$. Atmos. Sci., 49, 2183-2199.

Kubota, A. and M. Sugita, 1994: Radiometrically determined skin temperature and scalar roughness to estimate surface heat flux. Part I: Parameterization of radiometric scalar roughness. Bound.-Layer Meteor., 69, 397-416.

Kustas, W.P. and J.M. Norman, 1996: Use of remote sensing for evapotranspiration monitoring over land surfaces. Hydro. Sci. J., 41, 495-516.

— B.J. Choudhury, M.S. Moran, R.D.J.R.J. Reginato, L.W. Gay, and H.L. Weaver, 1989: Determination of sensible heat flux over sparse canopy using thermal infrared data. Agric. For. Meteor., 44, 197-216.

Lhomme, J.P., A. Chehbouni, and B. Monteny, 2000: Sensible heat flux-radiometric surface temperature relationship over sparse vegetation: $\mathrm{Pa}-$ rameterizing $B^{-1}$. Bound.-Layer Meteor., 97, 431-457.

Mahrt, L. and D. Vickers, 2004: Bulk formulation of the surface heat flux. Bound.-Layer Meteor., 110, 357-379.

—, J. Sun, J.I. MacPherson, N.O. Jensen, and R.L. Desjardins, 1997: Formation of surface heat flux: Application to BOREAS. J. Geophys. Res., 102, 29641-29649.

Matsushima, D. and J. Kondo, 1997: A proper method for estimating sensible heat flux above a horizontal-homogeneous vegetation canopy using radiometric surface observations. $J$. Appl. Meteor., 36, 1696-1711.

and - 2000: Estimating regional distribution of sensible heat flux over vegetation using satellite infrared temperature with viewing angle correction. J. Meteor. Soc. Japan, 78, 753-763.

Qualls, R.J. and D.N. Yates, 2001: Directional radiometric temperature profiles within a grass canopy. Adv. Wat. Resour., 24, 145-155.

Sugita, M. and W. Brutsaert, 1990: Regional surface fluxes from remotely sensed skin temperature 
and lower boundary layer measurements. Water Resour. Res., 26, 2937-2944.

Sun, J., 1999: Diurnal variations of thermal roughness height over a grassland. Bound.-Layer Meteor., 92, 407-427.

Troufleau, D., J.P. Lhomme, B. Monteny, and A. Videl, 1997: Sensible heat flux and radiometric surface temperature over sparse Sahelian vegetation. I An experimental analysis of the $k B^{-1}$ parameter. J. Hydro., 188-189, 815838.

Verhoef, A., H.A.R.D. Bruin, and B.J.J.M.V.D. Hurk, 1997: Some practical notes on the parameter $k B^{-1}$ for sparse vegetation. J. Appl. Meteor., 36, 560-572.

Vining, R.C. and B.L. Blad, 1992: Estimation of sensible heat flux from remotely sensed canopy temperatures. J. Geophys. Res., 97, 1895118954.

Voogt, J.A. and C.S.B. Grimmond, 2000: Modeling surface sensible heat flux using surface radiative temperatures in a simple urban area. $J$. Appl. Meteor., 39, 1679-1699. and T.R. Oke, 2003: Thermal remote sensing of urban climates. Remote Sens. Environ., 86, 370-384. 